\title{
Preparation of Iridium Oxide and its Application in Sensor-Actuator Systems
}

\author{
W. OLTHUIS, J. C. VAN KERKHOF and P. BERGVELD
}

Department of Electrical Engineering, University of Twente, P.O. Box 217, NL-7500 AE Enschede (The Netherlands)

M. BOS and W. E. VAN DER LINDEN

Department of Chemical Technology, University of Twente, P.O. Box 217, NL-7500 AE Enschede (The Netherlands)

\begin{abstract}
Acid or base concentrations can be determined by coulometric titration. A new alliridium oxide sensor-actuator device is presented, of which the titrant is generated by a reversible redox reaction occurring in the electroactive iridium oxide actuator electrode, and the equivalence point in the titration curve is detected by the $\mathrm{pH}$-sensitive iridium oxide sensor. The advantage with respect to the formerly used noble metal actuator electrode/ISFET sensor device is twofold: on the one hand, interference of $\mathrm{Cl}^{-}$ions, which oxidize at the noble metal actuator electrode during titration, is avoided and on the other hand, the preparation of the all-iridium oxide device is easier than of the ISFET-based devices. In addition to the conventional potential sweep method of oxide growth, a new current-pulse method is proposed, of which the parameters for optimal growth depend less on the $\mathrm{pH}$ of the solution.
\end{abstract}

\section{Introduction}

Acid or base concentrations can be determined by performing an acid-base titration with coulometrically generated $\mathrm{OH}^{-}$or $\mathrm{H}^{+}$ ions at a noble metal actuator electrode in close proximity to the $\mathrm{pH}$-sensitive gate of an ISFET. The ISFET is used as the indicator electrode to detect the equivalence point in the titration curve [1]. The equivalence time $t_{\text {eq }}$, that is the time needed to reach the equivalence point in the titration curve, depends on the acid or base concentration of the sample solution [2]. The potential of the

$0925-4005 / 91 / \$ 3.50$ noble metal actuator electrode during the generation of the titrant is that high for the anodic water electrolysis (or that low for the cathodic reaction) that other redox couples, possibly present in the sample solution, can interfere with the water electrolysis. This deteriorates the efficiency of the current to titrant generation on which this measurement relies.

To overcome this problem, iridium oxide is proposed as a new electroactive material for the actuator electrode [3]. The reversible redox reaction in this metal oxide occurs at a favourable potential and is attended with the exclusive uptake or release of protons, making a titration possible. A coulometric titration in the presence of $\mathrm{Cl}^{-}$ions, previously impossible with the noble metal actuator electrode because of the redox interference, can now successfully be carried out with iridium oxide as the actuator material.

Historically, the electrochemical growth of an oxide film on iridium was noticed during a triangular potential sweep, while recording a cyclic voltammogram. That is why triangular potential cycling has been the most widespread method of oxide growth, resulting in the so-called AIROF (anodic iridium oxide film) [4].

There are two inherent disadvantages of potential-controlled oxide growth. On the one hand, the limits of the potential sweeps are determined by cathodic and anodic water electrolysis and are, as such, $\mathrm{pH}$ dependent. Because these limits are critical for optimal growth, the $\mathrm{pH}$ of the electrolyte in which the oxide is formed has to be known fairly precisely. On the other hand, the actual formation of the oxide is related to the current

(C) Elsevier Sequoia/Printed in The Netherlands 


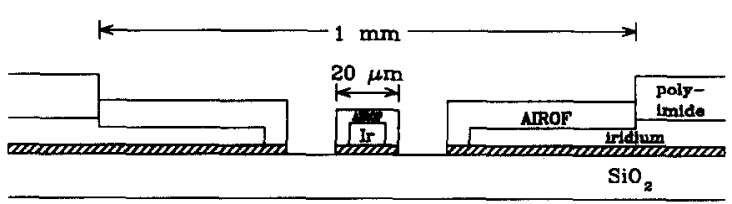

Si substrate

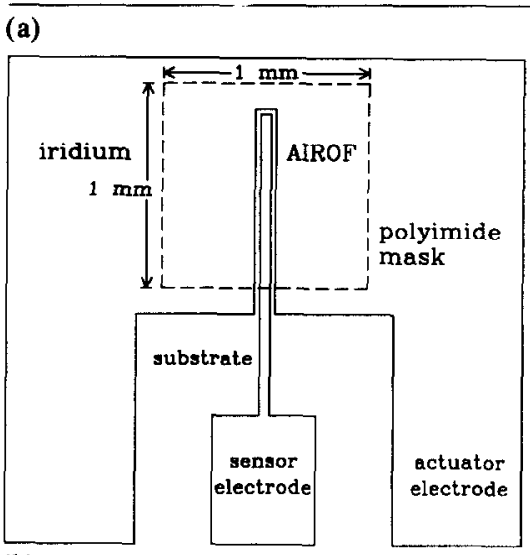

(b)

Fig. 1. (a) Cross section and (b) top vicw of the all-iridium oxide sensor-actuator device. Details of the preparation are discussed in the Experimental Section.

involved and as this current is not controlled (and may momentarily reach rather high peaks) the morphology of the oxide film may be undesirably affected. Therefore, in this paper a new method for forming iridium oxide is introduced, with which the current is the controlled parameter of the growth process.

Recently, the $\mathrm{pH}$-sensitive properties of iridium oxide have also been studied [5]. Iridium oxide turns out to be a suitable pH-sensitive electrode for rapid acid/base titrations. Hence, a device has been constructed of which both the actuator and the sensor consist of iridium oxide. This new all-iridium oxide sensor-actuator device is much easier to manufacture than the ISFET-based device. A cross section and top view of the device are given in Fig. 1.

\section{Experimental}

\section{Preparation}

An iridium film $\approx 100 \mathrm{~nm}$ thick was deposited by electron-gun evaporation on top of a $25 \mathrm{~nm} \mathrm{Ag}$ and $25 \mathrm{~nm} \mathrm{Ti}$ adhesion layer. These layers were evaporated on 2 inch p-Si wafers covered with $\approx 1 \mu \mathrm{m} \mathrm{SiO}{ }_{2}$. The iridium film was patterned using ion-beam etching, because a wet-chemical etchant for Ir is not known. As a mask, a photolithographically patterned polyimide layer of $\approx 1 \mu \mathrm{m}$ thickness was used. In this way structures with minimum lateral dimensions of $10 \mu \mathrm{m}$ could be obtained. A layer of polyimide was spun on the wafer with the patterned iridium film. In the layer, holes were etched to contact the sensor and the actuator electrode as well as to free the area of these electrodes to be exposed to the solution.

After dicing the wafer, the chips were glued onto a $1 \times 5 \mathrm{~cm}$ piece of printed circuit board and connected to the available copper strips with bonding wires. The copper strips, the bonding wires and the edges of the chip were covered with epoxy for insulation and protection.

The electrochemical growth of iridium oxide was carried out in a $0.5 \mathrm{M} \mathrm{H}_{2} \mathrm{SO}_{4}$ solution with a PAR $173 / 276$ potentiostat. The parameters that describe the triangular potential sweep applied to the iridium electrode are explained from Fig. 2(a). Both the upper potential limit $V_{u}$ and the lower potential limit $V_{1}$ play an important role in the oxide growth [6]. For the $0.5 \mathrm{M} \mathrm{H}_{2} \mathrm{SO}_{4}$ solution, the optimal potentials are $V_{\mathrm{u}}=1.25 \mathrm{~V}$ and $V_{1}=-0.25 \mathrm{~V}$ versus SCE. All potentials in this paper are presented relative to a saturated calomel electrode (SCE). These potential limits coincide with the onset of anodic and cathodic water electrolysis at an iridium electrode. It was concluded from the literature [6] that the rate of film growth is fairly constant if the sweep rate $v_{\mathrm{s}}(=\partial V / \partial t)$ is between 50 and $200 \mathrm{mV} / \mathrm{s}$. For the experiments described in this paper, $v_{\mathrm{s}}=150 \mathrm{mV} / \mathrm{s}$ was chosen. Hence, the total time for one triangular sweep is $t_{\mathrm{c}}=20 \mathrm{~s}$.

A new method of electrochemical oxide formation is introduced: current-pulse oxide growth. The criterion for successful electrochemical growth of an oxide layer and thus for the proper choice of the parameters $i_{\mathrm{a}}$ 


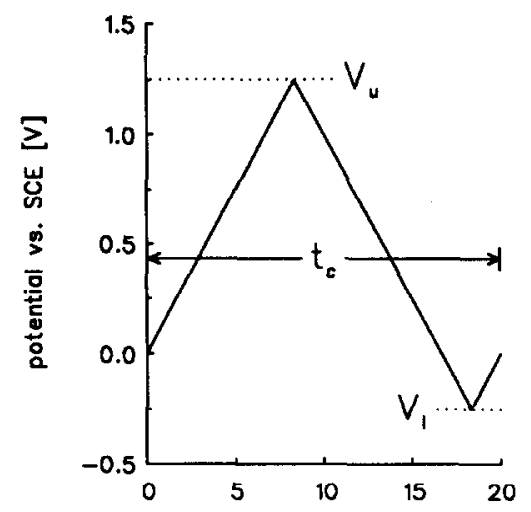

(a)

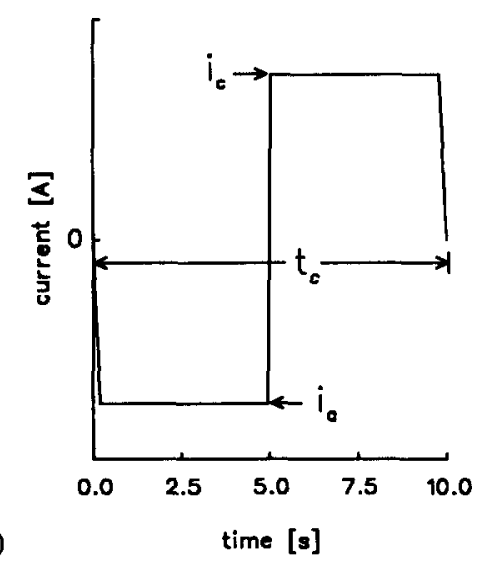

Fig. 2. (a) The triangular potential cycle causing oxide growth. The cycle is determined by the upper and lower potential limits $V_{\mathrm{u}}$ and $V_{\mathrm{l}}$, and by the scan rate $v_{\mathrm{s}}$ (fixing the cycle time $t_{\mathrm{c}}$ ). (b) The current pulse causing oxide growth. The pulse, and with that the amount of charge applied to the electrode, is determined by the currents $i_{\mathrm{a}}, i_{\mathrm{c}}$ and the cycle time $t_{\mathrm{c}}$.

$\left(=-i_{\mathrm{c}}\right)$ and $t_{\mathrm{c}}$, as given in Fig. 2(b), is the requirement that the potentials corresponding to anodic and cathodic water electrolysis are reached. This demand is fulfilled when a certain minimum amount of charge $\left|i_{\mathrm{a}} t_{\mathrm{c}} / 2\right|$ $\left(=i_{\mathrm{c}} t_{\mathrm{c}} / 2\right)$ causes both the maximum and the minimum electrode potential to flatten and reach a plateau, indicating that water electrolysis is reached. This flattening itself is enough indication for water electrolysis (and thus for oxide growth) and the value of the potential does not have to be known, making it easy to form oxide in a solution of which the $\mathrm{pH}$ is unknown.

The maximum current density during ox- ide growth was chosen not to exceed the current density that occurs during a coulometric titration, typically 10 to $50 \mu \mathrm{A} / \mathrm{mm}^{2}$ to avoid possible stress in the oxide film. The cycle time $t_{\mathrm{c}}$ was chosen to be typically between 2 and $20 \mathrm{~s}$. So the minimum and maximum limits of the charge applied $\left(\left|i_{\mathrm{a}} t_{\mathrm{c}} / 2\right|\right)$ are 10 to $500 \mu \mathrm{C} / \mathrm{mm}^{2}$. During the process of growth, the amount of charge that is exchanged has to be increased gradually as the oxide film thickens and the reversible redox reaction of the iridium oxide consumes more and more charge.

The iridium actuator and the iridium sensor were oxidized according to one of the two mentioned methods of oxide growth. Because the requirements for the actuator electrode oxide and the sensor oxide differ, they were oxidized separately: the actuator electrode oxide should be relatively thick in order to be able to exchange a certain minimum amount of charge (protons), necessary for the coulometric titration, whereas the sensor oxide should be kept relatively thin in order to obtain an acceptable response time to the $\mathrm{pH}$ change occurring during the coulometric titration.

\section{Measurement Set-up}

All measurements with the AIROF sensor-actuator device have been carried out with the set-up as shown in Fig. 3. The AIROF sensor was connected to an impedance converter with a very high input resistance $\left(>10^{12} \Omega\right)$. Apart from the AIROF sensor potential $V_{\text {AIRof,sensor }}$, the potential of the iridium oxide actuator, $V_{\text {AIROF, actuator }}$, was monitored during the titration in order to check whether the electrode potential stayed within the range determined by cathodic and anodic water electrolysis. All coulometric

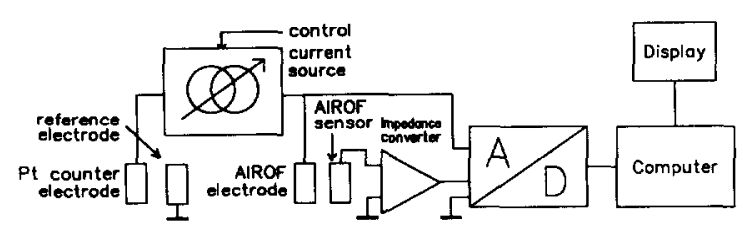

Fig. 3. Measurement set-up. 
titrations were carried out with an anodic or cathodic actuator electrode current density, $j_{\mathrm{a}}$ or $j_{\mathrm{c}}$, respectively, of $20 \mu \mathrm{A} / \mathrm{mm}^{2}$ in $0.1 \mathrm{M} \mathrm{KNO}_{3}$ supporting electrolyte.

\section{Results and Discussion}

\section{Characterization}

Figure 4 shows the result of a cyclic voltammogram of an AIROF electrode, recorded in $0.5 \mathrm{M} \mathrm{H}_{2} \mathrm{SO}_{4}$. The anodic and the cathodic peak are attended with the release and uptake of protons, respectively, according to the reaction $\mathrm{IrOOH} \rightleftharpoons \mathrm{IrO}_{2}+\mathrm{H}^{+}+e$ [4]. The potential at which these peaks are located, $0.7 \mathrm{~V}$, is well within the potentials at which cathodic and anodic water electrolysis occurs at a $\mathrm{Pt}$ electrode. The amount of protons that is exchanged, $\Delta Q$, can be estimated from the area of the cyclic voltammogram and for this electrode is $\approx 15 \mathrm{mC} / \mathrm{cm}^{2}$. The charge-exchange densities $\Delta Q$ for the device used in the titration experiments were $12 \mathrm{mC} / \mathrm{cm}^{2}$ for the actuator and $5 \mathrm{mC} / \mathrm{cm}^{2}$ for the sensor.

No difference in the electrochemical properties thus revealed was noticed between the potential sweep and the current-pulse method of oxide growth. The chemical stability, which was tested by storing the AIROFs for several days with and without applied potential sweeps in solutions with different $\mathrm{pH}$ and ionic composition, was comparable for both types of oxide growth; after $120 \mathrm{~h}$ of immersion in various electrolytes and 2400 applied triangular potential sweeps, $\Delta Q$ decayed by $\approx 18 \%$.

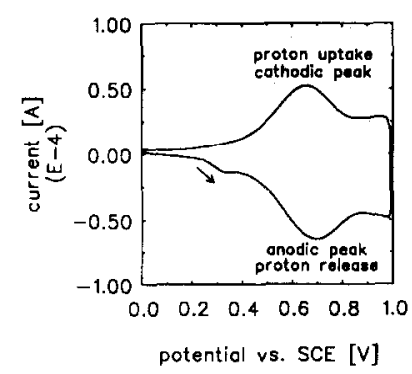

Fig. 4. Typical cyclic voltammogram of an electrochemically grown iridium oxide film in $0.5 \mathrm{M} \mathrm{H}_{2} \mathrm{SO}_{4}$, scan rate $=100 \mathrm{mV} / \mathrm{s}$.
In addition, current-pulse oxide growth was attempted in a $\mathrm{pH}=6.98$ buffer solution instead of the usual $0.5 \mathrm{M} \mathrm{H}_{2} \mathrm{SO}_{4}$ solution. There was no need to change any of the growth parameters $i_{\mathrm{a}}, i_{\mathrm{c}}$ or $t_{\mathrm{c}}$ (as given in Fig. 1(b)), because these parameters do not depend on the $\mathrm{pH}$ of the solution. After the formation, the oxide was characterized by cyclic voltammetry in $0.5 \mathrm{M} \mathrm{H}_{2} \mathrm{SO}_{4}$. There was no significant difference with the conventionally grown oxide, whose cyclic voltammogram is shown in Fig. 4.

\section{Typical Base to Acid Titration}

A typical base to acid titration, carried out in a $1 \mathrm{mM} \mathrm{KOH}$ sample, is shown in Fig. 5(a). This titration was started $1 \mathrm{~min}$ after the AIROF was totally charged with protons by reducing the film with a cathodic current of $10 \mu \mathrm{A} / \mathrm{mm}^{2}$ for $\approx 15 \mathrm{~s}$.

The potential span covered by the sensor signal $(-0.08$ to $0.42 \mathrm{~V}$ ) indicates a $\Delta \mathrm{pH} \approx 8$ and $t_{\text {eq }}=4.2 \mathrm{~s}$. At $t_{\mathrm{eq}}$, the actuator potential was $0.52 \mathrm{~V}$. This potential is almost $1 \mathrm{~V}$ lower
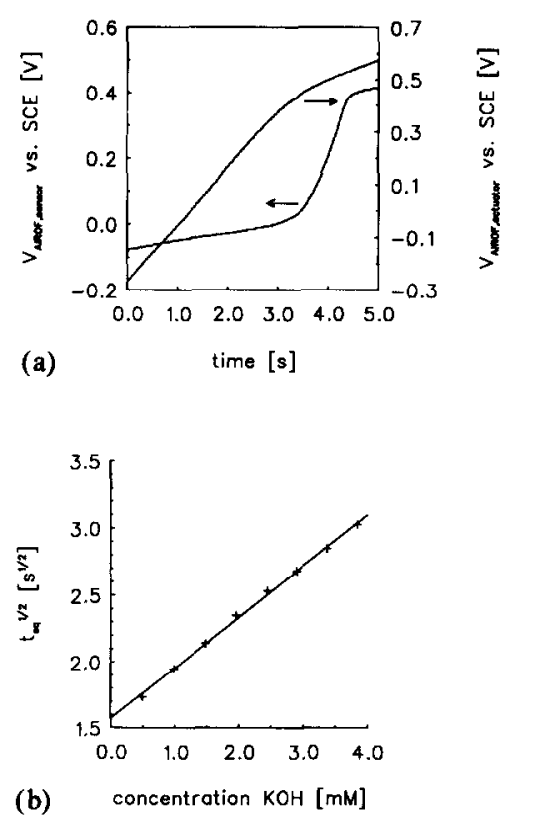

Fig. 5. (a) Typical base to acid titration carried out with an all-iridium oxide device in $1 \mathrm{mM} \mathrm{KOH}$; (b) square root of $t_{\text {eq }}$ as a function of the $\mathrm{KOH}$ bulk concentration measured with an all-iridium oxide device, $j_{\mathrm{a}}=20 \mathrm{~mA} / \mathrm{mm}^{2}$. 
than the potential of a noble metal actuator electrode during a comparable coulometric titration. This result offers favourable prospects concerning $\mathrm{Cl}^{-}$ion interference, which only occurs at an electrode potential of $\approx 1.4 \mathrm{~V}$.

To prove that neither the AIROF actuator behaviour nor the AIROF sensor signal were influenced by $\mathrm{Cl}^{-}$ions, $200 \mu$ l saturated $\mathrm{KCl}$ was added to the solution used for the previous experiment, resulting in a $50 \mathrm{mM} \mathrm{Cl}^{-}$ concentration. The coulometric titration was then repeated and both the sensor and the actuator potential were virtually equal to the curves of Fig. 5(a), as was $t_{\mathrm{eq}}(4.3 \mathrm{~s})$. It was thus shown that $\mathrm{Cl}^{-}$ions do not interfere at all up to the tested concentration of $50 \mathrm{mM}$.

\section{Determination of $\mathrm{KOH}$ Concentration}

A series of coulometric titrations with the all-iridium oxide device was carried out for eight different $\mathrm{KOH}$ concentrations. The reproducibility was good: the result of a second titration at each fixed concentration was within $2 \%$ of that of the first titration. The result is shown in Fig. 5(b).

The measurement result is in good agreement with the expected linear relation between the square root of $t_{\mathrm{eq}}$ and the $\mathrm{KOH}$ bulk concentration. The measured slope is about $22 \%$ larger $\left(0.38 \mathrm{~s}^{1 / 2} / \mathrm{mM}\right)$ than the expected slope [2] $\left(0.31 \mathrm{~s}^{1 / 2} / \mathrm{mM}\right)$. A possible explanation for this larger slope might be the observed loosening of small parts of the polyimide mask that determines the geometrical area of the actuator electrode. This increases the electrode area, thereby decreasing the actual current density $j_{\mathrm{a}}$, which increases the calculated slope.

\section{Typical Acid to Base Titration}

In Fig. 6(a) a typical acid to base titration carried out in a $2 \mathrm{mM}$ HAc sample solution is presented. This experiment is started $1 \mathrm{~min}$ after the actuator electrode was oxidized by an anodic current.

The initial and final sensor potentials indicate a successful titration: a change in potential of more than $0.5 \mathrm{~V}$ corresponds to a
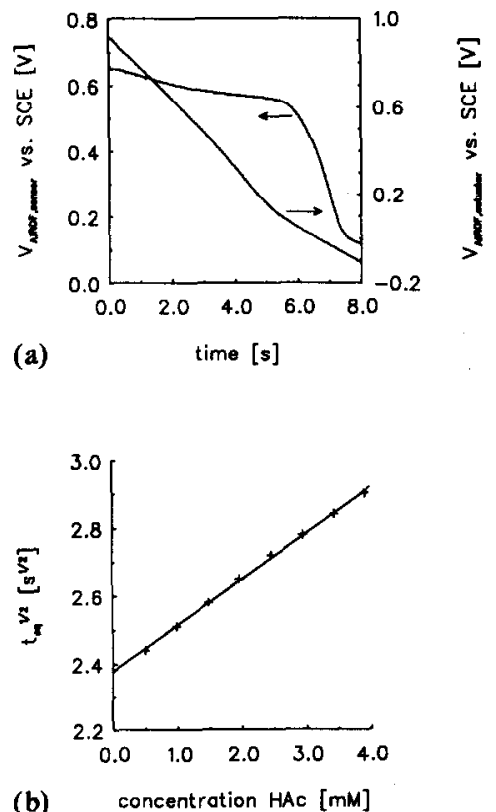

Fig. 6. (a) Typical acid to base titration carried out with an all-iridium oxide device in $2 \mathrm{mM} \mathrm{HAc}$; (b) square root of $t_{\text {eq }}$ as a function of the HAc bulk concentration measured with an all-iridium oxide device, $j_{\mathrm{c}}=20 \mathrm{~mA} / \mathrm{mm}^{2}$.

$\Delta \mathrm{pH} \approx 8$. The AIROF electrode potential at $t_{\mathrm{eq}}$ was $\approx 0.0 \mathrm{~V}$.

\section{Determination of HAc Concentration}

A series of coulometric titrations with the all-iridium oxide device was performed for eight different acetic acid concentrations. The reproducibility was good: the results of titrations carried out at a fixed HAc concentration did not differ more than $1 \%$. The result is presented in Fig. 6(b).

The measurement result is in good agreement with the expected linear relation between the square root of $t_{\text {eq }}$ and the HAc bulk concentration. The slope is about $9 \%$ smaller $\left(0.135 \mathrm{~s}^{1 / 2} / \mathrm{mM}\right)$ than the expected slope [2] $\left(0.149 \mathrm{~s}^{1 / 2} / \mathrm{mM}\right)$.

\section{Conclusions}

Coulometric acid-base titrations can successfully be accomplished with the new alliridium oxide sensor-actuator device even in 
the presence of $\mathrm{Cl}^{-}$ions, which interfere at the noble metal actuator electrode of an ISFET-based device.

The electrochemical formation of iridium oxide can either be accomplished by the conventional potential sweep of by the newly proposed current-pulse method of growth, for which the parameters controlling the growth are independent of the $\mathrm{pH}$ of the solution in which the oxide formation is accomplished.

\section{Acknowledgements}

This investigation is part of the research program of the Foundation for Fundamental Research on Matter (FOM), which is supported by the Netherlands Technology Foundation (STW).

\section{References}

1 W. Olthuis, B. H. van der Schoot, F. Chavez and P. Bergveld, A dipstick sensor for coulometric acid-base titrations, Sensors and Actuators, 17(1989) 279-283.

2 W. Olthuis, J. Luo, B. H. van der Schoot, J. G. Bomer and $P$. Bergueld, Dynamic behaviour of ISFET-based sensor-actuator systems, Sensors and Actuators $B, 1$ (1990) 416-420.

3 W. Olthuis, J. G. Bomer, P. Bergveld, M. Bos and W. E. van der Linden, Iridium oxide as actuator material for the ISFET-based sensor-actuator system, Proc. 3rd Int. Meet. Chemical Sensors, Cleveland, $\mathrm{OH}$, U.S.A., Sept. 1990.

4 W. Olthuis, M. A. M. Robben, P. Bergveld, M. Bos and $\mathbf{W}$. E. van der Linden, pH sensor properties of electrochemically grown iridium oxide, Sensors and Actuators B, 4 (1991) 247-256.

5 S. Gottesfeld and J. D. E. McIntyre, Electrochromism in anodic iridium oxide films, $J$. Electrochem. Soc., 126 (1979) $742-750$.

6 D. N. Buckley, L. D. Burke and J. K. Mulcahy, The oxygen electrode-part 5, J. Chem. Soc., Faraday Trans. 1, 71 (1975) 1447-1459. 